\title{
Seletividade de Glyphosate Isolado ou em Misturas PaRa SoJa RR EM APLicaÇões Sequenciais ${ }^{1}$
}

\author{
Selectivity of Glyphosate Alone or in Mixtures for RR Soybean in Sequential Applications
}

\author{
ALONSO, D.G. ${ }^{2}$, CONSTANTIN, J. ${ }^{3}$, OLIVEIRA JR., R.S. ${ }^{3}$, SANTOS, G. ${ }^{2}$, DAN, H.A. ${ }^{2}$ e \\ OLIVEIRA NETO, A.M. ${ }^{2}$
}

\begin{abstract}
RESUMO - O uso de misturas de herbicidas latifolicidas com o glyphosate tem sido uma alternativa para o controle de plantas daninhas resistentes ao glyphosate ou de dificil controle nos cultivos de soja RR. Este trabalho teve o objetivo de avaliar a seletividade de aplicações sequenciais de glyphosate, isolado ou em mistura com latifolicidas, para a soja RR. As aplicações dos tratamentos foram feitas em plantas de soja cultivar CD214 RR nos estádios de desenvolvimento V1 a V2 e V3 a V4. Os herbicidas e as respectivas doses (g i.a. ha ${ }^{-1}$ ) utilizadas foram: glyphosate (g e.a. ha-1) isolado $(720 / 480,720 / 720,960 / 960$ e 1.200/1.200) e as misturas de glyphosate + cloransulam-methyl $(720+15,12 / 480+15,12)$, glyphosate + fomesafen $(720+62,5 / 480+62,5)$, glyphosate + lactofen $(720+36 / 480+36)$, glyphosate + chlorimuron-ethyl $(720+6,25 / 480+6,25)$, glyphosate + flumiclorac-pentyl $(720+15 / 480+$ 15), glyphosate + bentazon $(720+240 / 480+240)$ e glyphosate + imazethapyr $(720+40 / 480+$ 40). O delineamento adotado foi o de blocos casualizados, em esquema de parcelas subdivididas, com quatro repetições. As variáveis avaliadas foram: fitointoxicação, altura de plantas, estande, fechamento do dossel, número de vagens por planta, massa de cem grãos e produtividade. Todos os tratamentos promoveram efeitos visuais de fitointoxicação na cultura da soja; todavia, em nenhuma das situações estudadas houve prejuizo no rendimento de grãos.
\end{abstract}

Palavras-chave: herbicidas, mistura em tanque, soja resistente ao glyphosate, tolerância

\begin{abstract}
The use of latifolicide mixed with glyphosate has been an alternative to control weeds resistant to glyphosate or difficult to control in RR soybean crops. The aim of this work was to evaluate the selectivity of sequential applications of glyphosate isolated or in mixture with latifolicide herbicides for $R R$ soybean. Treatment applications were carried out on the soybean cultivar plants $C D 214 R R$ at the developmental stages V1 up to V2 and V3 up to V4. The herbicides and respective rates ( $g$ a.i. ha-1) evaluated were: glyphosate (g a.e. ha ${ }^{1}$ ) alone (720/480, 720/720, 960/960, and $1.200 / 1.200)$ and the mixtures glyphosate + cloransulam-methyl $(720+15.12 / 480+15.12)$, glyphosate + fomesafen $(720+62.5 / 480+62.5)$, glyphosate + lactofen $(720+36 / 480+36)$, glyphosate + chlorimuron-ethyl $(720+6.25 / 480+6.25)$, glyphosate + flumiclorac-pentyl $(720+15 / 480+15)$, glyphosate + bentazon $(720+240 / 480+240)$, and glyphosate + imazethapyr $(720+40 / 480+40)$. The experimental design was completely randomized in a split-plot scheme with two checks, and four replicates. The variables analyzed were plant intoxication, plant height, stand,crop canopy, number of pods per plant, 100-grain mass, and yield. All the treatments promoted visual effects of plant intoxication on the soybean crop. However, yield loss was not observed in any of the situations studied.
\end{abstract}

Keywords: herbicides, tank mixture, glyphosate-resistant soybean, tolerance.

Recebido para publicação em 11.5.2011 e aprovado em 2.7.2012.

2 Doutorando do Programa de Pós-Graduação em Agronomia, Bolsista do CNPq - Brasil, Núcleo de Estudos Avançados em Ciência das Plantas Daninhas, Universidade Estadual de Maringá - NAPD/UEM, Av. Colombo 5790, 87020-900 Maringá-PR, $<$ alonsodg07@hotmail.com>; ${ }^{3}$ Professor Associado, D.Sc., Dep. de Agronomia, NAPD/UEM.

Planta Daninha, Viçosa-MG, v. 31, n. 1, p. 203-212, 2013 


\section{INTRODUÇÃO}

A resistência da soja ao herbicida glyphosate representou uma das maiores conquistas tecnológicas para a agricultura no final do último século. Essa nova tecnologia proporcionou aos agricultores uma nova ferramenta para o controle de plantas daninhas em pós-emergência, especialmente por possuir amplo espectro de ação. Entretanto, o uso de um mesmo mecanismo de ação (inibidor de EPSPs) em pós-emergência proporcionou rapidamente a seleção de biótipos de plantas daninhas de dificil controle ou resistentes.

Existem relatos de plantas daninhas resistentes ao glyphosate no Brasil e em várias áreas agrícolas do mundo (Lich et al., 1997; Norris et al., 2001; Norsworthy et al., 2001; Norsworthy \& Oliver, 2002; Ferreira et al., 2008; Carvalho et al., 2011). Atualmente, são reconhecidas 21 espécies resistentes ao glyphosate em regiões produtoras de todo o mundo (Weed Science, 2011).

A fim de reduzir a pressão de seleção de biótipos resistentes, o uso alternado de herbicidas com diferentes mecanismos de ação em aplicações isoladas ou misturas em tanque - apresenta-se como alternativa indispensável para o manejo de plantas daninhas na agricultura. O trabalho de Correia \& Durigan (2010) demonstra que a aplicação única ou sequencial de glyphosate não reduziu a produtividade da soja dos cultivares CD 214 RR e M-SOY 8008 RR; entretanto, a condução desse experimento não se deu na ausência de plantas daninhas (capinado durante todo o ciclo).

A adoção da aplicação sequencial de herbicidas vem se mostrando uma alternativa interessante para o controle de plantas daninhas, já que o parcelamento da dose acaba aumentando a seletividade dos herbicidas às culturas (Oliveira Jr. et al., 2006).

A aplicação sequencial de herbicidas pósemergentes é uma técnica que pode ser utilizada para possibilitar a ampliação do período efetivamente no limpo de que a cultura dispõe no início do ciclo. Uma vez que a maioria dos pós-emergentes empregados na cultura da soja possui apenas ação de contato, sem atividade residual no solo, a aplicação sequencial apresenta-se como uma alternativa para o controle de vários fluxos germinativos das plantas daninhas.

No entanto, a fim de que essa modalidade de aplicação seja viável, é usual parcelar a dose para que ela seja menor que a normalmente utilizada na aplicação única. Todavia, o somatório das aplicações pode implicar a utilização de uma dose semelhante ou superior à aplicação única.

Assim, o presente trabalho teve por objetivo avaliar a seletividade de glyphosate utilizado de forma isolada ou em misturas com outros herbicidas em aplicações sequenciais nos estádios V1 a V2 e V3 a V4 de plantas de soja do cultivar CD-214 RR.

\section{MATERIAL E MÉTODOS}

O ensaio foi instalado em área de plantio direto na Fazenda Nossa Senhora Aparecida, na cidade de Florai-PR, situada na latitude de $23^{\circ} 16^{\prime} 19,73^{\prime \prime}$ sul, longitude de 52 $23^{\prime} 04,30^{\prime \prime}$ oeste de Greenwich e altitude de $443 \mathrm{~m}$. O clima da região é caracterizado como subtropical, com chuvas de verão e invernos secos - Cfa, segundo a classificação de Köppen.

O solo da área experimental foi identificado como Latossolo Vermelho eutrófico LVe, textura arenosa, constituído por $36,0 \%$ de areia grossa, 54,0\% de areia fina, $8,0 \%$ de argila e 2,0\% de silte. Em sua análise química, em amostras retiradas de 0 a $20 \mathrm{~cm}$ de profundidade, ela apresentou $\mathrm{pH}$ de 5,7 em $\mathrm{CaCl}_{2}$; $2,36 \mathrm{cmol}_{\mathrm{c}} \mathrm{dm}^{-3} \mathrm{de} \mathrm{H}^{+}+\mathrm{Al}^{3+} ; 2,18 \mathrm{cmol}_{\mathrm{c}} \mathrm{dm}^{-3} \mathrm{de}$ $\mathrm{Ca}^{+2} ; 0,36 \mathrm{cmol}_{c} \mathrm{dm}^{-3} \mathrm{de} \mathrm{Mg}^{+2} ; 0,13 \mathrm{cmol}_{\mathrm{c}} \mathrm{dm}^{-3}$ de $\mathrm{K}^{+} ; 15,0 \mathrm{mg} \mathrm{dm}^{-3}$ de P; e 4,35 $\mathrm{g} \mathrm{dm}^{-3} \mathrm{de} \mathrm{C}_{\text {. }}$

A área em questão havia sido cultivada com aveia no inverno anterior à semeadura da soja, sendo esta dessecada dez dias antes da semeadura com 1.260 g e.a. ha ${ }^{-1}$ de glyphosate. Na semeadura, a adubação foi de $270 \mathrm{~kg} \mathrm{ha}{ }^{-1}$ da fórmula 0-18-18 e $83 \mathrm{~kg} \mathrm{ha}^{-1}$ de cloreto de potássio. As sementes foram tratadas com o fungicida Maxin (35 g i.a. $\mathrm{kg}^{-1}$ de Fludioxonil + 10 g i.a. kg-1 de Metalaxyl-M) na dose de $0,001 \mathrm{~L} \mathrm{~kg}^{-1}$ de sementes, com o inseticida Standak (250 g i.a. $\mathrm{L}^{-1}$ de Fipronil) na dose de $0,001 \mathrm{~L} \mathrm{~kg}^{-1}$ de sementes e também com inoculante turfoso na dose de $2 \mathrm{~g} \mathrm{~kg}^{-1}$ de sementes. Aos 40 dias após emergência, 
foram aplicados $83 \mathrm{~kg} \mathrm{ha}^{-1}$ de cloreto de potássio.

O cultivar utilizado no experimento foi o CD 214 RR, pertencente ao grupo de maturação precoce; a semeadura foi realizada dia 9/11/2006, com espaçamento de $0,45 \mathrm{~m}$ entre linhas e uma densidade de semeadura de aproximadamente 18 sementes por metro linear. A colheita foi feita manualmente, no dia 20/3/2007.

No experimento foi utilizado o delineamento experimental de blocos ao acaso, em esquema de parcelas subdivididas, utilizandose testemunhas duplas adjacentes, com quatro repetições. Os herbicidas foram os fatores estudados nas parcelas (tratamentos principais), e as condições de ausência ou de presença dos herbicidas foram os fatores estudados nas subparcelas. As subparcelas foram compostas por oito linhas de plantio espaçadas de $0,45 \mathrm{~m}$ e cinco metros de comprimento, totalizando $18 \mathrm{~m}^{2}$ por subparcela.

A área útil para as avaliações compreendeu as seis linhas centrais, desprezandose o meio metro inicial e final de cada subparcela. O experimento foi mantido livre da presença de plantas daninhas durante todo o período de execução, sendo realizadas capinas manuais sempre que necessário, de forma que não houvesse competição entre a soja e as plantas daninhas.

Uma técnica semelhante foi utilizada por Fagliari et al. (2001) para avaliação da seletividade de herbicidas em cana-de-açúcar. Mais recentemente, Meschede et al. (2004) e Jaremtchuk et al. (2008) também a utilizaram no estudo da seletividade de herbicidas na cultura da soja. Meschede et al. (2004) afirmaram que esse tipo de experimento confere maior controle da variabilidade do meio, especialmente quando se utiliza o tradicional delineamento em blocos casualizados, com uma única testemunha por bloco.

Os tratamentos com os herbicidas avaliados estão descritos na Tabela 1 . Nas aplicações, utilizou-se equipamento costal sob pressão constante de $2,0 \mathrm{kgf} \mathrm{cm}^{-2}$, pressurizado a $\mathrm{CO}_{2}$ e equipado com pontas tipo leque XR-110.02. Essas condições de aplicação proporcionaram o equivalente a $200 \mathrm{~L} \mathrm{ha}^{-1} \mathrm{de}$ volume de calda. Por ocasião das aplicações, as condições climáticas foram de $27{ }^{\circ} \mathrm{C}$ de temperatura e $73 \%$ de umidade relativa do ar, com o solo úmido e ventos de $4,0 \mathrm{~km} \mathrm{~h}^{-1}$, para a primeira aplicação, e de $34{ }^{\circ} \mathrm{C}$ de temperatura e $62 \%$ de umidade relativa do ar, com o solo úmido, na segunda aplicação. Os dados pluviométricos e de temperatura durante a

Tabela 1 - Tratamentos em mistura em tanque de glyphosate com herbicidas seletivos à soja em pós-emergência ou glyphosate isolado aplicados em sequencial, doses e épocas de aplicação utilizados no experimento. Floraí-PR, 2006/2007

\begin{tabular}{|l|c|c|}
\hline \multicolumn{1}{|c|}{ Tratamento } & $\begin{array}{c}\text { Dose } \\
\left(\mathrm{g} \mathrm{ha}^{-1}\right)^{1 /}\end{array}$ & $\begin{array}{c}\text { Épocas de aplicação } \\
\text { (estádio de desenvolvimento } \\
\text { da soja) }\end{array}$ \\
\hline Glyphosate+cloransulam-methyl/Glyphosate+cloransulam-methyl & $720+5,12 / 480+15,12$ & $\mathrm{~V}_{1}-\mathrm{V}_{2} / \mathrm{V}_{3}-\mathrm{V}_{4}$ \\
\hline Glyphosate+fomesafen/Glyphosate+fomesafen & $720+62,5 / 480+62,5$ & $\mathrm{~V}_{1}-\mathrm{V}_{2} / \mathrm{V}_{3}-\mathrm{V}_{4}$ \\
\hline Glyphosate+lactofen/Glyphosate+lactofen & $720+36 / 480+36$ & $\mathrm{~V}_{1}-\mathrm{V}_{2} / \mathrm{V}_{3}-\mathrm{V}_{4}$ \\
\hline Glyphosate+chlorimuron-ethyl/Glyphosate+chlorimuron-ethyl & $720+6,25 / 480+6,25$ & $\mathrm{~V}_{1}-\mathrm{V}_{2} / \mathrm{V}_{3}-\mathrm{V}_{4}$ \\
\hline Glyphosate+flumiclorac-pentyl/Glyphosate+flumiclorac-pentyl & $720+15 / 480+15$ & $\mathrm{~V}_{1}-\mathrm{V}_{2} / \mathrm{V}_{3}-\mathrm{V}_{4}$ \\
\hline Glyphosate+bentazon/Glyphosate+bentazon & $720+240 / 480+240$ & $\mathrm{~V}_{1}-\mathrm{V}_{2} / \mathrm{V}_{3}-\mathrm{V}_{4}$ \\
\hline Glyphosate+imazethapyr/Glyphosate+imazethapyr & $720+40 / 480+40$ & $\mathrm{~V}_{1}-\mathrm{V}_{2} / \mathrm{V}_{3}-\mathrm{V}_{4}$ \\
\hline Glyphosate/Glyphosate & $720 / 480$ & $\mathrm{~V}_{1}-\mathrm{V}_{2} / \mathrm{V}_{3}-\mathrm{V}_{4}$ \\
\hline Glyphosate/Glyphosate & $720 / 720$ & $\mathrm{~V}_{1}-\mathrm{V}_{2} / \mathrm{V}_{3}-\mathrm{V}_{4}$ \\
\hline Glyphosate/Glyphosate & $960 / 960$ & $\mathrm{~V}_{1}-\mathrm{V}_{2} / \mathrm{V}_{3}-\mathrm{V}_{4}$ \\
\hline Glyphosate/Glyphosate & $1.200 / 1.200$ & $\mathrm{~V}_{1}-\mathrm{V}_{2} / \mathrm{V}_{3}-\mathrm{V}_{4}$ \\
\hline
\end{tabular}

1/ gramas de ingrediente ativo ou equivalente ácido por hectare. ²/ V3 a V4 realizada 15 dias após a primeira aplicação. +: Misturas em tanque. /: Aplicações sequenciais. 
realização do experimento são apresentados na Figura 1.

Foram realizadas avaliações visuais de fitointoxicação, altura das plantas, estande, fechamento das entrelinhas e contagem do número de vagens por planta. Após a colheita, foram avaliadas a massa de cem grãos e a produtividade.

Nas avaliações visuais de fitointoxicação, foram atribuídas notas percentuais a cada unidade experimental que recebeu os herbicidas $(0$, para ausência de injúrias, até $100 \%$, para morte das plantas), considerando-se, nesse caso, os sintomas visíveis nas plantas de acordo com seu desenvolvimento (SBCPD, 1995), aos 3, 7 e 15 dias após a primeira aplicação (DAA) e aos três dias após a segunda aplicação (DASA).

Avaliações visuais do fechamento da cultura foram realizadas aos 15 DAA e aos 3 e 15 DASA, a fim de determinar o efeito dos tratamentos no desenvolvimento do dossel da cultura. A escala utilizada foi a de notas percentuais de zero (entrelinha descoberta) até $100 \%$, representando o fechamento total da entrelinha.

O estande foi avaliado para verificar se houve interferência das aplicações de herbicidas na população de plantas de soja. Foi feita a contagem do número de plantas vivas aos 15 DASA em três metros lineares em duas linhas centrais das parcelas, obtendo-se uma média do número de plantas por metro linear.
Para determinação da altura média das plantas $(\mathrm{cm})$, mediu-se a altura de dez plantas por parcela, obtendo-se uma média. As plantas foram medidas ao acaso dentro da área útil de cada unidade experimental. A medida foi feita do solo até a inserção do último trifolíolo completamente expandido, aos 15 e 90 DASA.

Durante a colheita, foram feitas contagens de vagens em dez plantas por parcela, as quais foram retiradas ao acaso das áreas úteis, obtendo-se a média por planta de cada unidade experimental. As áreas úteis de cada unidade experimental foram colhidas manualmente, sendo posteriormente trilhadas, ensacadas e pesadas.

A massa de cem grãos foi obtida por meio da contagem de três amostras de cem grãos por unidade experimental, as quais foram posteriormente pesadas. Também foram retiradas três amostras de grãos por parcela, para determinação da umidade e correção das massas obtidas para a umidade de $13 \%$.

Os dados obtidos foram submetidos à análise de variância pelo teste $\mathrm{F}$, e as médias, comparadas pelo teste de Tukey a $5 \%$ de probabilidade.

\section{RESULTADOS E DISCUSSÃO}

\section{Fitointoxicação}

Os dados referentes às avaliações visuais de fitointoxicação (porcentagem de injúrias)

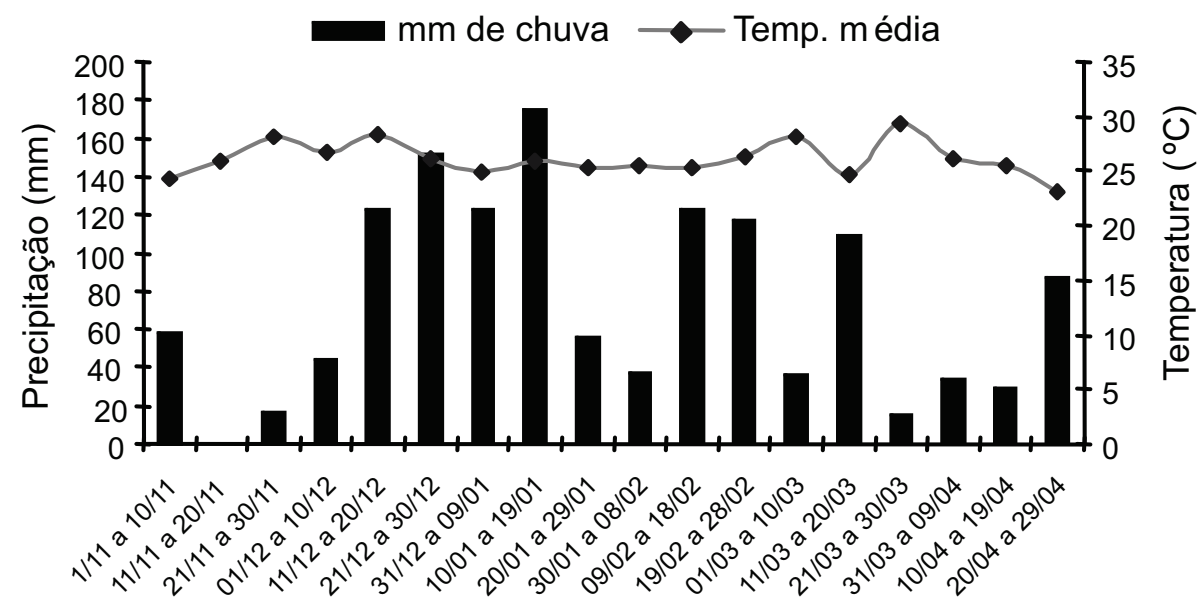

Figura 1 - Valores médios decendiais de temperatura média $\left({ }^{\circ} \mathrm{C}\right)$ e precipitação (mm) no período de execução do experimento. FloraíPR, 2006/2007. 
realizadas após as aplicações dos herbicidas encontram-se na Tabela 2.

Pode-se observar nos referidos resultados que todos os tratamentos promoveram injúrias visuais à cultura da soja. Aos 3 e 7 DAA, os sintomas observados demonstram que doses reduzidas dos herbicidas seletivos convencionais podem provocar fortes injúrias quando misturadas com glyphosate; glyphosate + lactofen, glyphosate + chlorimuron-ethyl, glyphosate + fomesafen e glyphosate + bentazon foram as misturas mais prejudiciais à cultura nesse período, promovendo índices de fitointoxicação de 65, 65, 40 e 40\%, respectivamente, aos 7 DAA.

Maciel et al. (2009) verificaram níveis de intoxicação de 17,8 e 25,5\% para o cultivar CD $214 \mathrm{RR}$ aos 7 DAA, para as misturas de glyphosate + chlorimuron-ethyl nas doses de $720+7,5$ e $720+10,0\left(\mathrm{~g} \mathrm{ha}^{-1}\right)$, respectivamente, aplicadas no estádio V3-V4 da cultura.

Alonso et al. (2010) demonstraram que misturas de herbicidas pós-emergentes com glyphosate para soja tolerante a esse herbicida apresentam maior potencial de fitointoxicação para aplicações em estádios mais precoces de desenvolvimento da cultura. Entretanto, segundo esses autores, no caso da necessidade de misturas de herbicidas para complementação do espectro de ação do glyphosate, é preferivel que as misturas sejam usadas em aplicações mais precoces dentro do ciclo da cultura, visto que, com aplicação de misturas na primeira aplicação sequencial entre V1 e V2, a cultura terá maior período de tempo para se recuperar das injúrias provocadas pelos tratamentos.

Tabela 2 - Média das notas de fitointoxicação (\%) em soja, cultivar CD 214 RR, submetida a diferentes tratamentos sequenciais com glyphosate isolado ou em mistura com outros herbicidas em pós-emergência. Floraí-PR, 2006/2007

\begin{tabular}{|c|c|c|c|c|c|}
\hline \multirow{3}{*}{ Herbicidas } & \multirow{3}{*}{$\begin{array}{l}\text { Dose } \\
\left(\mathrm{g} \mathrm{ha}^{-1}\right)^{1 /}\end{array}$} & \multicolumn{4}{|c|}{ Fitointoxicação (\%) } \\
\hline & & \multicolumn{3}{|c|}{$1^{\mathrm{a}}$ aplicação } & \multirow{2}{*}{$\frac{2^{\mathrm{a}} \text { aplicação }}{3 \text { DASA }}$} \\
\hline & & $3 \mathrm{DAA}$ & 7 DAA & $15 \mathrm{DAA}$ & \\
\hline $\begin{array}{l}\text { Glyphosate+cloransulam-methyl/ } \\
\text { Glyphosate+cloransulam-methyl }\end{array}$ & $\begin{array}{l}720+15,12 / \\
480+15,12\end{array}$ & 35,0 & 30,0 & 11,3 & 45,0 \\
\hline $\begin{array}{l}\text { Glyphosate+fomesafen/ } \\
\text { Glyphosate+fomesafen }\end{array}$ & $\begin{array}{l}720+62,5 / \\
480+62,5\end{array}$ & 50,0 & 40,0 & 25,0 & 50,0 \\
\hline $\begin{array}{l}\text { Glyphosate+lactofen/ } \\
\text { Glyphosate+lactofen }\end{array}$ & $\begin{array}{l}720+36 / \\
480+36\end{array}$ & 60,0 & 65,0 & 35,0 & 65,0 \\
\hline $\begin{array}{l}\text { Glyphosate+chlorimuron-ethyl/ } \\
\text { Glyphosate+chlorimuron-ethyl }\end{array}$ & $\begin{array}{l}720+6,25 / \\
480+6,25\end{array}$ & 45,0 & 65,0 & 30,0 & 61,3 \\
\hline $\begin{array}{l}\text { Glyphosate+flumiclorac-pentyl/ } \\
\text { Glyphosate+flumiclorac-pentyl }\end{array}$ & $\begin{array}{l}720+15 / \\
480+15\end{array}$ & 35,0 & 35,0 & 15,0 & 40,0 \\
\hline $\begin{array}{l}\text { Glyphosate+bentazon/ } \\
\text { Glyphosate+bentazon }\end{array}$ & $\begin{array}{l}720+240 / \\
480+240\end{array}$ & 50,0 & 40,0 & 15,0 & 25,0 \\
\hline $\begin{array}{l}\text { Glyphosate+imazethapyr/ } \\
\text { Glyphosate+imazethapyr }\end{array}$ & $\begin{array}{l}720+40 / \\
480+40\end{array}$ & 42,5 & 31,3 & 15,0 & 36,3 \\
\hline $\begin{array}{l}\text { Glyphosate/ } \\
\text { Glyphosate }\end{array}$ & $\begin{array}{l}720 / \\
480\end{array}$ & 20,0 & 25,0 & 10,0 & 25,0 \\
\hline $\begin{array}{l}\text { Glyphosate/ } \\
\text { Glyphosate }\end{array}$ & $\begin{array}{l}720 / \\
720\end{array}$ & 20,0 & 35,0 & 5,0 & 30,0 \\
\hline $\begin{array}{l}\text { Glyphosate/ } \\
\text { Glyphosate }\end{array}$ & $\begin{array}{l}960 / \\
960\end{array}$ & 25,0 & 32,5 & 10,0 & 33,8 \\
\hline $\begin{array}{l}\text { Glyphosate/ } \\
\text { Glyphosate }\end{array}$ & $\begin{array}{l}1.200 / \\
1.200\end{array}$ & 35,0 & 40,0 & 10,0 & 30,0 \\
\hline
\end{tabular}

1/ gramas de ingrediente ativo ou equivalente ácido por hectare. DAA: dias após a aplicação; DASA = dias após a segunda aplicação. (\%): 0 $\%=$ nenhum dano, $100 \%=$ morte das plantas. +: Misturas em tanque. /: Aplicações sequenciais. 
Outro fator relevante é que aplicações tanto de glyphosate isolado como em misturas são mais eficientes no controle de plantas daninhas de dificil controle (E. heterophylla e I. grandifolia) no estádio de uma a três folhas (Ramires et al., 2010).

Aos 15 DAA, os sintomas de fitointoxicação se tornaram menos intensos, ficando evidentes apenas nas folhas mais velhas. Já aos três dias após a segunda aplicação, os dados revelam que os tratamentos ocasionaram nova intoxicação às plantas, causando novo estresse no momento em que elas estavam se recuperando da primeira aplicação. Após esse período, não foram verificados novos sintomas, e os sintomas existentes desapareceram gradativamente com o desenvolvimento da cultura. Em condições adversas de desenvolvimento, como falta de água ou deficit de nutrientes no solo, esses sintomas podem persistir por um período maior, ocasionando perdas significativas de produtividade.

Embora o glyphosate isolado tenha proporcionado elevados índices de fitointoxicação, estes foram inferiores ou no máximo semelhantes aos das misturas menos tóxicas à cultura. Os sintomas apresentados por glyphosate foram caracterizados por leve clorose em muitas folhas.

\section{Avaliação visual do fechamento das entrelinhas pela cultura}

A Tabela 3 contém os resultados das avaliações visuais realizadas em relação ao fechamento do dossel da soja, aos 15 DAA e aos 3 e 15 DASA.

Comparando as aplicações sequenciais das misturas glyphosate + cloransulam-methyl, glyphosate + bentazon ou glyphosate isolado nas doses de $720 / 480$ e $720 / 720 \mathrm{~g} \mathrm{ha}^{-1}$ com suas respectivas testemunhas, observa-se que esses tratamentos não afetaram o desenvolvimento do dossel da cultura em todas as avaliações realizadas.

Aplicações sequenciais de glyphosate isolado nas doses de 1.200/1.200 $\mathrm{g} \mathrm{ha}^{-1}$ ou em misturas com fomesafen ou imazethapyr causaram reduções significativas no fechamento da cultura aos 3 DASA. Todavia, aos 15 DASA, as plantas já haviam se recuperado.
Quanto aos tratamentos glyphosate + flumiclorac-pentyl e glyphosate $\left(960 / 960 \mathrm{~g} \mathrm{ha}^{-1}\right)$, observa-se que houve atraso no fechamento das entrelinhas nas avaliações de 15 dias após a primeira aplicação e aos 3 DASA.

O tratamento com aplicações sequenciais de glyphosate + lactofen alterou o desenvolvimento vegetativo das plantas aos 3 e 15 DASA, sugerindo que os efeitos negativos da primeira aplicação somados aos da segunda aplicação da mistura afetaram significativamente o desenvolvimento da cultura.

Quando comparado o fechamento das entrelinhas que receberam o tratamento sequencial contendo glyphosate + chlorimuronethyl com a sua respectiva testemunha sem herbicida, nota-se que em todas as avaliações as plantas tratadas foram afetadas pela mistura, evidenciando forte efeito supressor no desenvolvimento das plantas desse tratamento.

As misturas de glyphosate + chlorimuron, glyphosate + flumiclorac e glyphosate/ glyphosate $\left(960 / 960 \mathrm{~g} \mathrm{ha}^{-1}\right)$ continuaram a suprimir o crescimento do dossel até a avaliação de 3 DASA; contudo, apenas misturas contendo chlorimuron ou lactofen afetaram o fechamento até 15 DASA. Esses efeitos estão associados aos níveis de fitointoxicação observados para esses tratamentos, nos quais fortes injúrias reduziram a área foliar das plantas.

De modo geral, observou-se que aos 3 DASA a maioria dos tratamentos prejudicou o fechamento das entrelinhas. Esses resultados devem-se ao efeito de fitotoxicidade promovido pela aplicação sequencial, aliado ao estresse inicial causado pela primeira aplicação, o que paralisou temporariamente o desenvolvimento da cultura da soja.

A avaliação de fechamento das entrelinhas é uma ferramenta importante a ser considerada em um trabalho de seletividade com culturas anuais, pois, quanto menor a influência do herbicida sobre a cultura, mais rapidamente o dossel sombreará as entrelinhas, reduzindo assim a emergência das plantas daninhas fotoblásticas positivas. 
Tabela 3 - Fechamento da soja, cultivar CD 214 RR, submetida a diferentes tratamentos sequenciais com glyphosate isolado ou em mistura com outros herbicidas em pós-emergência, aos 15 dias após a primeira aplicação (DAA) e aos 3 e 15 dias após a segunda aplicação (DASA). Floraí-PR, 2006/2007

\begin{tabular}{|c|c|c|c|c|c|c|c|}
\hline \multirow[t]{2}{*}{ Herbicidas } & \multirow{2}{*}{$\begin{array}{c}\text { Dose } \\
\left(\mathrm{g} \mathrm{ha}^{-1}\right)^{\underline{1 /}}\end{array}$} & \multicolumn{2}{|c|}{$\begin{array}{c}\text { Fechamento } \\
\text { (15 DAA) } \\
\text { Herbicida }^{2 /}\end{array}$} & \multicolumn{2}{|c|}{$\begin{array}{c}\text { Fechamento } \\
\text { (3 DASA) } \\
\text { Herbicida }^{2 /}\end{array}$} & \multicolumn{2}{|c|}{$\begin{array}{r}\text { Fechamento } \\
\left(15 \text { DASA }^{2}\right. \\
\text { Herbicida }^{2 /}\end{array}$} \\
\hline & & Com & Sem & Com & Sem & Com & Sem \\
\hline $\begin{array}{l}\text { Glyphosate+cloransulam-methy/ } \\
\text { Glyphosate+cloransulam-methyl }\end{array}$ & $\begin{array}{l}720+15,12 / \\
480+15,12\end{array}$ & $38,75 \mathrm{a}$ & $39,00 \mathrm{a}$ & $55,00 \mathrm{a}$ & $55,63 \mathrm{a}$ & $95,50 \mathrm{a}$ & $95,25 \mathrm{a}$ \\
\hline $\begin{array}{l}\text { Glyphosate+fomesafen/ } \\
\text { Glyphosate+fomesafen }\end{array}$ & $\begin{array}{l}720+62,5 / \\
480+62,5\end{array}$ & $37,50 \mathrm{a}$ & $43,75 \mathrm{a}$ & $50,00 \mathrm{~b}$ & $68,75 \mathrm{a}$ & $95,25 \mathrm{a}$ & $97,00 \mathrm{a}$ \\
\hline $\begin{array}{l}\text { Glyphosate+lactofen/ } \\
\text { Glyphosate+lactofen }\end{array}$ & $\begin{array}{l}720+36 / \\
480+36\end{array}$ & $41,25 \mathrm{a}$ & $47,50 \mathrm{a}$ & $51,25 \mathrm{~b}$ & $68,13 \mathrm{a}$ & $87,75 \mathrm{~b}$ & 96,25 a \\
\hline $\begin{array}{l}\text { Glyphosate+chlorimuron-ethyl/ } \\
\text { Glyphosate+chlorimuron-ethyl }\end{array}$ & $\begin{array}{l}720+6,25 / \\
480+6,25\end{array}$ & $32,50 \mathrm{~b}$ & $40,63 \mathrm{a}$ & $50,00 \mathrm{~b}$ & $63,13 \mathrm{a}$ & $92,00 \mathrm{~b}$ & $97,13 \mathrm{a}$ \\
\hline $\begin{array}{l}\text { Glyphosate+flumiclorac-pentyl/ } \\
\text { Glyphosate+flumiclorac-pentyl }\end{array}$ & $\begin{array}{l}720+15 / \\
480+15\end{array}$ & $28,75 \mathrm{~b}$ & $36,88 \mathrm{a}$ & $45,00 \mathrm{~b}$ & $58,75 \mathrm{a}$ & $93,25 \mathrm{a}$ & $96,38 \mathrm{a}$ \\
\hline $\begin{array}{l}\text { Glyphosate+bentazon/ } \\
\text { Glyphosate+bentazon }\end{array}$ & $\begin{array}{l}720+240 / \\
480+240 \\
\end{array}$ & $38,75 \mathrm{a}$ & $38,75 \mathrm{a}$ & $53,75 \mathrm{a}$ & $57,50 \mathrm{a}$ & $96,25 \mathrm{a}$ & 96,38 a \\
\hline $\begin{array}{l}\text { Glyphosate+imazethapyr/ } \\
\text { Glyphosate+imazethapyr }\end{array}$ & $\begin{array}{l}720+40 / \\
480+40\end{array}$ & $30,00 \mathrm{a}$ & $36,88 \mathrm{a}$ & $46,25 \mathrm{~b}$ & $58,75 \mathrm{a}$ & $93,75 \mathrm{a}$ & 96,50 a \\
\hline $\begin{array}{l}\text { Glyphosate/ } \\
\text { Glyphosate }\end{array}$ & $\begin{array}{l}720 / \\
480 \\
\end{array}$ & $40,00 \mathrm{a}$ & $44,38 \mathrm{a}$ & $61,25 \mathrm{a}$ & $63,13 \mathrm{a}$ & $98,00 \mathrm{a}$ & $98,00 \mathrm{a}$ \\
\hline $\begin{array}{l}\text { Glyphosate/ } \\
\text { Glyphosate }\end{array}$ & $\begin{array}{l}720 / \\
720\end{array}$ & 33,75 a & $36,88 \mathrm{a}$ & $53,75 \mathrm{a}$ & $60,00 \mathrm{a}$ & $95,00 \mathrm{a}$ & 96,13 a \\
\hline $\begin{array}{l}\text { Glyphosate/ } \\
\text { Glyphosate }\end{array}$ & $\begin{array}{l}960 / \\
960\end{array}$ & $28,75 \mathrm{~b}$ & $36,88 \mathrm{a}$ & $45,00 \mathrm{~b}$ & $58,13 \mathrm{a}$ & $96,25 \mathrm{a}$ & 96,88 a \\
\hline $\begin{array}{l}\text { Glyphosate/ } \\
\text { Glyphosate }\end{array}$ & $\begin{array}{l}1.200 / \\
1.200\end{array}$ & $34,25 \mathrm{a}$ & $37,75 \mathrm{a}$ & $47,50 \mathrm{~b}$ & $59,38 \mathrm{a}$ & $94,25 \mathrm{a}$ & 96,50 a \\
\hline CV $(\%)$ & & \multicolumn{2}{|c|}{13,80} & \multicolumn{2}{|c|}{8,71} & \multicolumn{2}{|c|}{2,33} \\
\hline DMS (Tukey, 5\%) & & \multicolumn{2}{|c|}{7,44} & \multicolumn{2}{|c|}{7,01} & \multicolumn{2}{|c|}{3,20} \\
\hline
\end{tabular}

Para cada variável analisada, médias seguidas das mesmas letras na mesma linha (com x sem herbicida) não diferem entre si pelo teste de Tukey a $5 \%$ de probabilidade. ${ }^{1 /}$ gramas de ingrediente ativo ou equivalente ácido por hectare. ${ }^{2 /}$ Representam parcelas que receberam aplicações de herbicidas (COM) e suas respectivas testemunhas adjacentes que não receberam tratamento herbicida (SEM). +: Misturas em tanque. /: Aplicações sequenciais.

\section{Altura e estande de plantas}

Os valores médios de altura $(\mathrm{cm})$ aos 15 e 90 DASA e do estande de plantas (plantas $\mathrm{m}^{-1}$ ) de soja aos 15 DASA estão representados na Tabela 4.

Os dados apresentados demonstram que a aplicação sequencial de glyphosate + chlorimuron-ethyl reduziu significativamente a altura das plantas aos 15 DASA, porém elas se recuperaram posteriormente (90 DASA). Nesse caso, os efeitos observados na altura e fechamento das plantas são condizentes (Tabela 3), comprovando o efeito supressor do desenvolvimento promovido por esse tratamento.

A aplicação sequencial de glyphosate (960 / $960 \mathrm{~g} \mathrm{ha}^{-1}$ ) provocou redução significativa na altura das plantas aos 90 DASA.

No que concerne à aplicação sequencial de glyphosate + lactofen, os resultados sugerem que esse tratamento foi extremamente prejudicial ao crescimento da soja, pois nas duas avaliações de altura de plantas os tratamentos apresentaram-se inferiores à testemunha sem herbicida. É possivel que não tenha havido tempo suficiente para a recuperação das plantas, ficando assim com seu 
Tabela 4 - Altura média (cm) aos 15 e 90 dias após a segunda aplicação (DASA) e estande (plantas m-1) aos 15 DASA das plantas de soja, cultivar CD $214 \mathrm{RR}$, submetida a diferentes tratamentos sequenciais com glyphosate isolado ou em mistura com outros herbicidas em pós-emergência. Floraí-PR, 2006/2007

\begin{tabular}{|c|c|c|c|c|c|c|c|}
\hline \multirow{3}{*}{ Herbicida } & \multirow{3}{*}{$\begin{array}{l}\text { Doses } \\
\left(\mathrm{g} \mathrm{ha}^{-1}\right)^{\frac{1}{}}\end{array}$} & \multirow{2}{*}{\multicolumn{2}{|c|}{$\begin{array}{c}\text { Altura (15 DASA) } \\
\text { Herbicida }^{2 /}\end{array}$}} & \multirow{2}{*}{\multicolumn{2}{|c|}{$\frac{\text { Altura (90 DASA) }}{\text { Herbicida }^{2}{ }^{2 /}}$}} & \multirow{2}{*}{\multicolumn{2}{|c|}{$\frac{\text { Estande (15 DASA) }}{\text { Herbicida }^{\underline{2}}}$}} \\
\hline & & & & & & & \\
\hline & & Com & Sem & Com & Sem & Com & Sem \\
\hline $\begin{array}{l}\text { Glyphosate+cloransulam-methyl/ } \\
\text { Glyphosate+cloransulam-methyl }\end{array}$ & $\begin{array}{l}720+15,12 / \\
480+15,12\end{array}$ & $32,45 \mathrm{a}$ & $32,49 \mathrm{a}$ & $48,53 \mathrm{a}$ & $53,11 \mathrm{a}$ & $14,00 \mathrm{a}$ & $15,29 \mathrm{a}$ \\
\hline $\begin{array}{l}\text { Glyphosate+fomesafen/ } \\
\text { Glyphosate+fomesafen }\end{array}$ & $\begin{array}{l}720+62,5 / \\
480+62,5\end{array}$ & $34,80 \mathrm{a}$ & $36,96 \mathrm{a}$ & $49,83 \mathrm{a}$ & $56,20 \mathrm{a}$ & $14,71 \mathrm{a}$ & $14,67 \mathrm{a}$ \\
\hline $\begin{array}{l}\text { Glyphosate+lactofen/ } \\
\text { Glyphosate+lactofen }\end{array}$ & $\begin{array}{l}720+36 / \\
480+36\end{array}$ & $29,08 \mathrm{~b}$ & $35,68 \mathrm{a}$ & $44,43 \mathrm{~b}$ & $55,59 \mathrm{a}$ & $15,21 \mathrm{a}$ & $14,31 \mathrm{a}$ \\
\hline $\begin{array}{l}\text { Glyphosate+chlorimuron-ethyl/ } \\
\text { Glyphosate+chlorimuron-ethyl }\end{array}$ & $\begin{array}{l}720+6,25 / \\
480+6,25\end{array}$ & $32,45 \mathrm{~b}$ & 38,36 a & $49,05 \mathrm{a}$ & $53,65 \mathrm{a}$ & $14,92 \mathrm{a}$ & $15,15 \mathrm{a}$ \\
\hline $\begin{array}{l}\text { Glyphosate+flumiclorac-pentyl/ } \\
\text { Glyphosate+flumiclorac-pentyl }\end{array}$ & $\begin{array}{l}720+15 / \\
480+15\end{array}$ & $29,23 \mathrm{a}$ & $34,30 \mathrm{a}$ & $45,75 \mathrm{a}$ & $50,10 \mathrm{a}$ & 14,17 a & $15,08 \mathrm{a}$ \\
\hline $\begin{array}{l}\text { Glyphosate+bentazon/ } \\
\text { Glyphosate+bentazon }\end{array}$ & $\begin{array}{l}720+240 / \\
480+240 \\
\end{array}$ & $35,83 \mathrm{a}$ & $35,21 \mathrm{a}$ & $49,15 \mathrm{a}$ & $51,63 \mathrm{a}$ & $15,29 \mathrm{a}$ & $15,65 \mathrm{a}$ \\
\hline $\begin{array}{l}\text { Glyphosate+imazethapyr/ } \\
\text { Glyphosate+imazethapyr }\end{array}$ & $\begin{array}{l}720+40 / \\
480+40 \\
\end{array}$ & $29,25 \mathrm{a}$ & 33,44 a & $46,95 \mathrm{a}$ & $50,91 \mathrm{a}$ & $14,58 \mathrm{a}$ & $14,65 \mathrm{a}$ \\
\hline $\begin{array}{l}\text { Glyphosate/ } \\
\text { Glyphosate }\end{array}$ & $\begin{array}{l}720 / \\
480\end{array}$ & $36,15 \mathrm{a}$ & $38,96 \mathrm{a}$ & $56,78 \mathrm{a}$ & $59,10 \mathrm{a}$ & $15,33 \mathrm{a}$ & $15,40 \mathrm{a}$ \\
\hline $\begin{array}{l}\text { Glyphosate/ } \\
\text { Glyphosate }\end{array}$ & $\begin{array}{l}720 / \\
720\end{array}$ & $34,35 \mathrm{a}$ & $36,80 \mathrm{a}$ & $52,60 \mathrm{a}$ & $54,13 \mathrm{a}$ & $14,38 \mathrm{a}$ & $14,44 \mathrm{a}$ \\
\hline $\begin{array}{l}\text { Glyphosate/ } \\
\text { Glyphosate }\end{array}$ & $\begin{array}{l}960 / \\
960\end{array}$ & $32,45 \mathrm{a}$ & $36,94 \mathrm{a}$ & $46,50 \mathrm{~b}$ & $55,40 \mathrm{a}$ & $14,63 \mathrm{a}$ & $15,71 \mathrm{a}$ \\
\hline $\begin{array}{l}\text { Glyphosate/ } \\
\text { Glyphosate }\end{array}$ & $\begin{array}{l}1200 / \\
1200\end{array}$ & $29,73 \mathrm{a}$ & $34,60 \mathrm{a}$ & $51,08 \mathrm{a}$ & $53,94 \mathrm{a}$ & $16,38 \mathrm{a}$ & $15,58 \mathrm{a}$ \\
\hline CV $(\%)$ & & \multicolumn{2}{|c|}{10,68} & \multicolumn{2}{|c|}{9,57} & \multicolumn{2}{|c|}{7,63} \\
\hline DMS (Tukey, 5\%) & & \multicolumn{2}{|c|}{5,24} & \multicolumn{2}{|c|}{7,11} & \multicolumn{2}{|c|}{1,64} \\
\hline
\end{tabular}

Para cada variável analisada, médias seguidas das mesmas letras na mesma linha (com x sem herbicida) não diferem entre si pelo teste de Tukey a $5 \%$ de probabilidade. ${ }^{1 /}$ gramas de ingrediente ativo ou equivalente ácido por hectare. ${ }^{2 /}$ Representam parcelas que receberam aplicações de herbicidas (COM) e suas respectivas testemunhas adjacentes que não receberam tratamento herbicida (SEM). +: Misturas em tanque. /: Aplicações sequenciais.

crescimento vegetativo comprometido após a segunda aplicação. Em seu trabalho, Almeida Jr. et al. (2010) também observaram que as misturas de glyphosate + chlorimuron e glyphosate + lactofen reduziram significativamente a altura das plantas.

Todos os demais tratamentos não apresentaram diferenças significativas quando comparados com suas testemunhas duplas adjacentes. Com relação à altura de plantas, o efeito da aplicação sequencial de herbicidas está relacionado ao cultivar de soja, pois, sob condições ambientais semelhantes, um mesmo tratamento pode ser prejudicial à altura de plantas para uma variedade e indiferente para a outra (Maciel et al., 2009).
Quando comparados os estandes das testemunhas com os dos tratamentos, verifica-se que, apesar de os tratamentos sequenciais terem afetado de alguma forma o desenvolvimento das plantas (altura e fechamento), essas injúrias não foram suficientes para provocar reduções no estande da cultura.

\section{Número de vagens por planta, massa de cem grãos e produtividade}

Na Tabela 5 são apresentados os dados referentes a número de vagens por planta, massa de cem grãos e produtividade de grãos.

O único tratamento que afetou de forma negativa o número de vagens por planta de soja 
foi o glyphosate $\left(720 / 720 \mathrm{~g} \mathrm{ha}^{-1}\right)$. No entanto, esse efeito não se repetiu para as maiores doses (960/960 e 1.200/1.200 $\left.\mathrm{g} \mathrm{ha}^{-1}\right)$.

Quanto ao efeito dos tratamentos no que se refere à massa média de cem grãos, as misturas de glyphosate + cloransulam-methyl, glyphosate + lactofen e glyphosate (1.200/ $1.200 \mathrm{~g} \mathrm{ha}^{-1}$ ) afetaram significativamente essa variável. Os demais tratamentos não afetaram significativamente a massa de cem grãos de soja.

A avaliação da produtividade de soja para este experimento demonstrou que, apesar dos efeitos negativos provocados por alguns tratamentos no desenvolvimento vegetativo das plantas (altura e fechamento) ou mesmo no desenvolvimento reprodutivo (vagens por planta e massa de cem grãos), em nenhum caso esses efeitos causaram injúrias suficientes a ponto de reduzir o rendimento de grãos. Resultados semelhantes foram obtidos por Correia et al. (2008), nos quais misturas de glyphosate com lactofen e glyphosate com flumioxazin promoveram sintomas severos de fitointoxicação, como necroses e pontos cloróticos nas folhas; contudo, ainda assim, esses efeitos não se refletiram no desenvolvimento vegetativo e reprodutivo da soja.

Contrariamente, Alonso et al. (2010) verificaram que os tratamentos glyphosate/

Tabela 5 - Número médio de vagens por planta, massa de cem grãos (g) e produtividade (kg ha-1) de soja cultivar CD 214 RR, submetida a diferentes tratamentos seqUenciais com glyphosate isolado ou em mistura com outros herbicidas em pós-emergência. Floraí-PR, 2006/2007

\begin{tabular}{|c|c|c|c|c|c|c|c|}
\hline \multirow{3}{*}{ Herbicida } & \multirow{3}{*}{$\begin{array}{l}\text { Dose } \\
\left(\mathrm{g} \mathrm{ha}^{-1}\right)^{\frac{1}{}}\end{array}$} & \multirow{2}{*}{\multicolumn{2}{|c|}{$\begin{array}{c}\begin{array}{c}\mathrm{N}^{\mathrm{o}} \text { vagens } \\
\text { (vagens por planta) }\end{array} \\
\text { Herbicida }^{\underline{2}}\end{array}$}} & \multirow{2}{*}{\multicolumn{2}{|c|}{$\begin{array}{c}\begin{array}{c}\text { Massa de cem grãos } \\
(\mathrm{g})\end{array} \\
\text { Herbicida }^{2 /}\end{array}$}} & \multirow{2}{*}{\multicolumn{2}{|c|}{$\begin{array}{c}\begin{array}{c}\text { Produtividade } \\
\left(\mathrm{kg} \mathrm{ha}^{-1}\right)\end{array} \\
\text { Herbicida }^{2 /} \\
\end{array}$}} \\
\hline & & & & & & & \\
\hline & & Com & Sem & Com & Sem & Com & Sem \\
\hline $\begin{array}{l}\text { Glyphosate+cloransulam-methyl/ } \\
\text { glyphosate+cloransulam-methyl }\end{array}$ & $\begin{array}{l}720+15,12 / \\
480+15,12\end{array}$ & $47,75 \mathrm{a}$ & $45,39 \mathrm{a}$ & $11,78 \mathrm{~b}$ & $12,28 \mathrm{a}$ & $3.020,71 \mathrm{a}$ & $2.928,29 \mathrm{a}$ \\
\hline $\begin{array}{l}\text { Glyphosate+fomesafen/ } \\
\text { glyphosate+fomesafen }\end{array}$ & $\begin{array}{l}720+62,5 / \\
480+62,5\end{array}$ & $44,23 \mathrm{a}$ & $51,30 \mathrm{a}$ & $11,92 \mathrm{a}$ & $12,36 \mathrm{a}$ & $2.804,48 \mathrm{a}$ & $2.989,29 \mathrm{a}$ \\
\hline $\begin{array}{l}\text { Glyphosate+lactofen/ } \\
\text { glyphosate+lactofen }\end{array}$ & $\begin{array}{l}720+36 / \\
480+36\end{array}$ & 48,70 a & $51,56 \mathrm{a}$ & $11,20 \mathrm{~b}$ & $12,08 \mathrm{a}$ & $2.743,12 \mathrm{a}$ & $2.810,58 \mathrm{a}$ \\
\hline $\begin{array}{l}\text { Glyphosate+chlorimuron-ethyl/ } \\
\text { glyphosate+chlorimuron-ethyl }\end{array}$ & $\begin{array}{l}720+6,25 / \\
480+6,25\end{array}$ & $44,05 \mathrm{a}$ & 46,16 a & $12,06 \mathrm{a}$ & $12,09 \mathrm{a}$ & $2.859,57 \mathrm{a}$ & $2.885,69 \mathrm{a}$ \\
\hline $\begin{array}{l}\text { Glyphosate+flumiclorac-pentyl/ } \\
\text { glyphosate+flumiclorac-pentyl }\end{array}$ & $\begin{array}{l}720+15 / \\
480+15\end{array}$ & 50,83 a & 46,75 a & $12,30 \mathrm{a}$ & $12,50 \mathrm{a}$ & $2.745,47 \mathrm{a}$ & $2.899,92 \mathrm{a}$ \\
\hline $\begin{array}{l}\text { Glyphosate+bentazon/ } \\
\text { glyphosate+bentazon }\end{array}$ & $\begin{array}{l}720+240 / \\
480+240 \\
\end{array}$ & $39,20 \mathrm{a}$ & 44,58 a & $12,14 \mathrm{a}$ & $12,11 \mathrm{a}$ & $2.801,63 \mathrm{a}$ & $2.828,35 \mathrm{a}$ \\
\hline $\begin{array}{l}\text { Glyphosate+imazethapyr/ } \\
\text { glyphosate+imazethapyr }\end{array}$ & $\begin{array}{l}720+40 / \\
480+40\end{array}$ & $47,20 \mathrm{a}$ & $46,85 \mathrm{a}$ & $12,08 \mathrm{a}$ & $12,34 \mathrm{a}$ & $2.752,44 \mathrm{a}$ & $2.883,02 \mathrm{a}$ \\
\hline $\begin{array}{l}\text { Glyphosate/ } \\
\text { Glyphosate }\end{array}$ & $\begin{array}{l}720 / \\
480 \\
\end{array}$ & $49,10 \mathrm{a}$ & 49,84 a & $12,43 \mathrm{a}$ & $12,61 \mathrm{a}$ & $3.132,22 \mathrm{a}$ & $3.111,73 \mathrm{a}$ \\
\hline $\begin{array}{l}\text { Glyphosate/ } \\
\text { Glyphosate }\end{array}$ & $\begin{array}{l}720 / \\
720 \\
\end{array}$ & $42,68 \mathrm{~b}$ & 52,73 a & $11,84 \mathrm{a}$ & $12,22 \mathrm{a}$ & $2.713,53 \mathrm{a}$ & $2.951,82 \mathrm{a}$ \\
\hline $\begin{array}{l}\text { Glyphosate/ } \\
\text { Glyphosate }\end{array}$ & $\begin{array}{l}960 / \\
960 \\
\end{array}$ & $49,85 \mathrm{a}$ & 49,33 a & $12,25 \mathrm{a}$ & $12,25 \mathrm{a}$ & $2.831,23 \mathrm{a}$ & $3.074,49 \mathrm{a}$ \\
\hline $\begin{array}{l}\text { Glyphosate/ } \\
\text { Glyphosate }\end{array}$ & $\begin{array}{l}1200 / \\
1200\end{array}$ & $43,58 \mathrm{a}$ & $45,86 \mathrm{a}$ & $11,40 \mathrm{~b}$ & 12,11 a & $2.793,70 \mathrm{a}$ & $2.900,75 \mathrm{a}$ \\
\hline $\mathrm{CV}(\%)$ & & \multicolumn{2}{|c|}{13,42} & \multicolumn{2}{|c|}{2,79} & \multicolumn{2}{|c|}{6,14} \\
\hline DMS (Tukey, 5\%) & & \multicolumn{2}{|c|}{9,11} & \multicolumn{2}{|c|}{0,49} & \multicolumn{2}{|c|}{254,84} \\
\hline
\end{tabular}

Para cada variável analisada, médias seguidas das mesmas letras na mesma linha (com x sem herbicida) não diferem entre si pelo teste de Tukey a $5 \%$ de probabilidade. ${ }^{1 /}$ gramas de ingrediente ativo ou equivalente ácido por hectare. ${ }^{2} /$ Representam parcelas que receberam aplicações de herbicidas (COM) e suas respectivas testemunhas adjacentes que não receberam tratamento herbicida (SEM). +: misturas em tanque. /: aplicações sequenciais. 
glyphosate + lactofen $\left(720 / 480+72 \mathrm{~g} \mathrm{ha}^{-1}\right)$ e glyphosate/glyphosate + bentazon (720/ $480+480 \mathrm{~g} \mathrm{ha}^{-1}$ ), aplicados nos estádios V1-V2/V3-V4, promoveram efeitos negativos na produtividade da soja (CD $214 \mathrm{RR}$ ).

Esse fato evidencia que o parcelamento da dose em aplicações sequenciais pode ser benéfico no que concerne aos efeitos prejudiciais dos herbicidas à cultura da soja. Assim, conclui-se que todos os tratamentos foram seletivos ao cultivar CD $214 \mathrm{RR}$, para as condições em que este estudo foi realizado.

\section{LITERATURA CITADA}

ALMEIDA JR., J. J. et al. Utilização de glyphosate associado a herbicidas em pós-emergência na cultura da soja geneticamente modificada. R. Bras. Herbic., v. 9, n. 3, p. 100-108, 2010.

ALONSO, D. G. et al. Selectivity of glyphosate tank mixtures for RR soybean in sequential applications with mixtures only in the first or second application. Planta Daninha, v. 28, n. 4 , p. $865-875,2010$.

CARVALHO, L. B. et al. Detection of sourgrass (Digitaria insularis) biotypes resistant to glyphosate in Brazil. Weed Sci., v. 59, n. 2, p. 171-176, 2011.

CORREIA, N. M.; DURIGAN, J. C. Controle de plantas daninhas na cultura de soja resistente ao glyphosate.

Bragantia, v. 69, n. 2, p. 319-329, 2010.

CORREIA, N. M.; DURIGAN, J. C.; LEITE, G. J. Seletividade da soja transgênica tolerante ao glyphosate e eficácia de controle de Commelina benghalensis com herbicidas aplicados isolados e em misturas. Bragantia, v. 67, n. 3, p. 663-671, 2008.

FAGLIARI, J.R.; OLIVEIRA JR., R.S.; CONSTANTIN, J. Métodos de avaliação da seletividade de herbicidas para a cultura da cana-de-açúcar (Saccharum spp.). Acta Sci. Agron., v. 23, n. 5, p. 1229-1234, 2001.

FERREIRA, E. A. et al. Distribuição de glyphosate e acúmulo de nutrientes em biótipos de azevém. Planta Daninha, v. 26, n. 1, p. 165-173, 2008.
JAREMTCHUK, C. C. et al. Efeito de sistemas de manejo sobre a velocidade de dessecação, infestação inicial de plantas daninhas e desenvolvimento e produtividade de soja. Acta Sci. Agron., v. 30, n. 4, p. 449-455, 2008.

LICH, J. M.; RENNER, K. A.; PENNER, D. Interaction of glyphosate with postemergence soybean (Glycine max) herbicides. Weed Sci., v. 45, n. 1, p. 12-21, 1997.

MESCHEDE, D. K. et al. Período anterior à interferência de plantas daninhas em soja: estudo de caso com baixo estande e testemunhas duplas. Planta Daninha, v. 22, n. 2, p. 239-246, 2004

MACIEL, C. D. G. et al. Seletividade de cultivares de soja $\mathrm{RR}^{\circledR}$ submetidos a misturas em tanque de glyphosate + chlorimuron-ethyl associadas a óleo mineral e inseticidas Planta Daninha, v. 27, n. 4, p. 755-768, 2009.

NORRIS, J. L.; SHAW, D. R.; SNIPES, C. E. Weed control from herbicide combinations with three formulations of glyphosate. Weed Technol., v. 15, n. 3, p. 552-558, 2001.

NORSWORTHY, J. K.; BURGOS, N. R.; OLIVER, L. R. Differences in weed tolerance to glyphosate involve different mechanisms. Weed Technol., v. 15, n. 4, p. 725-731, 2001.

NORSWORTHY, J. K.; OLIVER, L. R. Pitted morningglory interference in drill-seeded glyphosate-tolerant soybean. Weed Sci., v. 50, n. 1, p. 26-33, 2002.

OLIVEIRA JR., R. S. et al. Aplicações sequenciais de flumiclorac-pentil para o controle de Euphorbia heterophylla na cultura da soja. Acta Sci. Agron., v. 28, n. 1, p. 115-122, 2006.

RAMIRES, A. C. et al. Controle de Euphorbia heterophylla e Ipomoea grandifolia com a utilização de glyphosate isolado ou em associação com latifolicidas. Planta Daninha, v. 28, n. 3, p. 621-629, 2010.

SOCIEDADE BRASILEIRA DA CIÊNCIA DAS PLANTAS DANINHAS - SBCPD. Procedimentos para instalação, avaliação e análise de experimentos com herbicidas. Londrina: $1995.42 \mathrm{p}$.

WEED SCIENCE - INTERNATIONAL SURVEY OF HERBICIDE RESISTANT WEEDS. Disponível em: <http:// www.weedscience.org > . Acesso em: 15 set. 2011. 